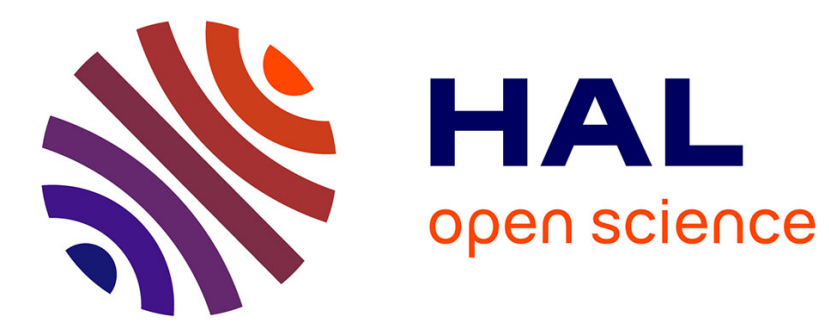

\title{
Morse theory and Lyapunov stability on manifolds Emmanuel Moulay
}

\section{To cite this version:}

Emmanuel Moulay. Morse theory and Lyapunov stability on manifolds. Journal of Mathematical Sciences, 2011, 177 (3), pp.419-425. 10.1007/s10958-011-0468-6 . hal-00655923

\section{HAL Id: hal-00655923 https://hal.science/hal-00655923}

Submitted on 19 Jan 2021

HAL is a multi-disciplinary open access archive for the deposit and dissemination of scientific research documents, whether they are published or not. The documents may come from teaching and research institutions in France or abroad, or from public or private research centers.
L'archive ouverte pluridisciplinaire $\mathbf{H A L}$, est destinée au dépôt et à la diffusion de documents scientifiques de niveau recherche, publiés ou non, émanant des établissements d'enseignement et de recherche français ou étrangers, des laboratoires publics ou privés. 


\title{
Morse theory and Lyapunov stability on manifolds
}

\author{
E. Moulay \\ Xlim (UMR-CNRS 6172) - Département SIC \\ Université de Poitiers - Bât. SP2MI \\ Bvd Marie et Pierre Curie - BP 30179 \\ 86962 Futuroscope Chasseneuil Cedex - France
}

\begin{abstract}
The aim of this article is to recall the main theorems of Morse theory and to infer some corollaries for the problem of Lyapunov stability on manifolds. It makes a link between Morse theory and the general theory of the Lyapunov stability for dynamical systems.
\end{abstract}

Keywords: Morse theory, Lyapunov stability, Lyapunov functions.

\section{Introduction}

The main goal of this paper is to see the implications of Morse theory for the problem of Lyapunov stability on manifolds. Indeed, stability and stabilization for nonlinear systems evolving on manifolds are a new research field (see for instance [1]). In differential topology, Morse theory developed by John Milnor in the twentieth century gives a very direct way of analyzing the topology of a manifold by studying differentiable functions on that manifold. It has also been used and mentioned in some papers dedicated to Lyapunov stability in control theory (see $[1,2,3,4])$. Nevertheless, a paper that links Morse theory and Lyapunov stability in control theory has never been addressed in the literature.

The paper is organized as follows. Some preliminaries are outlined in Section 2. In Section 3, we analyze the Morse Lyapunov functions. In Section 4, we consider the first Morse theorem known as the deformation lemma and its application for dynamical systems with asymptotically stable sets. In Section 5 , we recall the Brown-Stallings lemma which leads to a necessary condition of asymptotic stability and the second Morse theorem dedicated to dynamical systems with a single critical point. We show why this second Morse theorem is not really accurate in control theory due to a more precise result proved by Sontag in [3]. We provide the third Morse Theorem with the Reeb Theorem and their applications for stability of dynamical systems with multi critical points in Section 6. Finally, Morse inequalities lead to a necessary condition for the existence of a Morse Lyapunov function.

Email address: emmanuel.moulay@univ-poitiers.fr (E. Moulay) 


\section{Notations and definitions}

In the paper, $\mathcal{M}$ denotes a manifold of finite dimension $n$. Consider a continuous vector field $f$ on $\mathcal{M}$ with the property that for every $x \in \mathcal{M}$, there exists a unique right maximally defined integral curve of $f$ starting at $x$, and, furthermore, every right maximally defined integral curve of $f$ is defined on $[0,+\infty)$. In this case, the integral curves of $f$ are jointly continuous functions of time and initial condition and thus define a continuous semi-flow

$$
\varphi:[0,+\infty) \times \mathcal{M} \rightarrow \mathcal{M}
$$

satisfying

$$
\begin{aligned}
\varphi(0, x) & =x \\
\varphi\left(t_{1}, \varphi\left(t_{2}, x\right)\right) & =\varphi\left(t_{1}+t_{2}, x\right)
\end{aligned}
$$

for all $t_{1}, t_{2} \in[0,+\infty)$ and $x \in \mathcal{M}$ (see for instance [5, Theorem 3.4]). The manifold $\mathcal{M}$, called the state space, and the semi flow $\varphi$, called the evolution function, lead to the notion of dynamical system denoted in short by $(\mathcal{M}, \varphi)$ (see for instance [5] and [6] for a general definition of topological dynamical systems).

$T_{p} \mathcal{M}$ denotes the tangent space to $\mathcal{M}$ at $p$.

In a general way, we study the stability of invariant sets.

Definition 1. Let $\mathcal{I} \subset \mathcal{M}, \mathcal{I}$ is an invariant set if for all $x \in \mathcal{I}$ and $t \in \mathbb{R}_{\geq 0}$, $\varphi(t, x) \in \mathcal{I}$. If $\mathcal{I}$ is a point, $\mathcal{I}$ is called an equilibrium point of the dynamical system $(\mathcal{M}, \varphi)$.

Let us recall the notions of stability given in [6].

Definition 2. $\mathcal{I} \subset \mathcal{M}$ is stable if every open neighborhood $\mathcal{U}_{1} \subset \mathcal{M}$ of $\mathcal{I}$, there exist an open neighborhood $\mathcal{U}_{2} \subset \mathcal{M}$ of $\mathcal{I}$ such that $\varphi\left(t, \mathcal{U}_{2}\right) \subseteq \mathcal{U}_{1}$ for all $t \geq 0$, where

$$
\varphi(t, \mathcal{U})=\{\varphi(t, x): x \in \mathcal{U}\}
$$

An invariant set $\mathcal{I}$ is asymptotically stable if:

- I is stable,

- I is attractive: i.e. for all $x \in \mathcal{I}$ there exist an open neighborhood $\mathcal{N} \subset \mathcal{M}$ of $\mathcal{I}$ such that for all $x \in \mathcal{N}, \varphi(t, x) \rightarrow \mathcal{I}$ as $t+\infty$.

The domain of attraction is denoted by

$$
\mathcal{A}=\{x \in \mathcal{M}: \varphi(t, x) \rightarrow \mathcal{I} \text { as } t \rightarrow+\infty\} .
$$

Besides, $\mathcal{I}$ is globally asymptotically stable if $\mathcal{N}=\mathcal{M}$.

The lie derivative of $V: \mathcal{M} \rightarrow \mathbb{R}$ along $f: \mathcal{M} \rightarrow \mathcal{M}$ is defined by

$$
\mathcal{L}_{f} V: \mathbb{R}^{n} \rightarrow \mathbb{R}, \quad \mathcal{L}_{f} V(x)=d V_{p}(f(p)) .
$$


Definition 3. Suppose that $\mathcal{K}$ is an invariant set of the dynamical system $(\mathcal{M}, \varphi)$. A continuous function $V: \mathcal{A} \rightarrow \mathbb{R}_{\geq 0}$ is a Lyapunov function if

1. $V(x)>0$ for all $x \in \mathcal{A} \backslash \mathcal{K}$,

2. $V(x)=0$ for all $x \in \mathcal{K}$,

3. $V$ is proper, that is, $V^{-1}(B)$ is compact for every compact subset $B$ of $\mathbb{R}_{>0}$,

4. $V$ is strictly decreasing along orbits of $\varphi$, that is, $V(\varphi(t, x))<V(x)$ for all $t>0$ and $x \in \mathcal{A} \backslash \mathcal{K}$.

If $V$ is differentiable, Condition 4 is replaced by $\mathcal{L}_{f} V(x)<0$ for all $x \in \mathcal{A} \backslash \mathcal{K}$.

We recall some definitions of Morse theory given in [7].

Definition 4. Let $V: \mathcal{M} \rightarrow \mathbb{R}$ be a smooth function. A critical point of $V$ is a point $p \in \mathcal{M}$ at which the differential

$$
d V_{p}: T_{p} \mathcal{M} \rightarrow T_{V(p)} \mathbb{R}
$$

has rank zero, i.e. if in any local coordinate system $\left(x_{1}, \ldots, x_{n}\right)$ around $p$ one has

$$
\left(\frac{\partial V}{\partial x_{1}}(p), \ldots, \frac{\partial V}{\partial x_{n}}(p)\right)=0 .
$$

For each critical point $p$ of $V$, there exists a bilinear symmetric form $H_{p}(V)$ on $T_{p} \mathcal{M}$, called the Hessian of $p$ (see [7, Chapter 2]).

Definition 5. A critical point $p$ is a non-degenerate critical point if the Hessian $H_{p}(V)$ is a non-degenerate bilinear form, i.e. if in any local coordinate system $\left(x_{1}, \ldots, x_{n}\right)$ around $p$, the Hessian matrix $\left(\frac{\partial^{2} V}{\partial x_{i} \partial x_{j}}(p)\right)_{1 \leq i, j \leq n}$ is nondegenerate.

The dimension of the subspace of $T_{p} \mathcal{M}$ on which $H_{p}(V)$ is negative definite is called the Morse index of $V$ at $p$ and is denoted by ind $(V, p)$.

$A C^{2}$ function $V: \mathcal{M} \rightarrow \mathbb{R}$ is a Morse function if all its critical points are non-degenerate.

The level sets of a function $V: \mathcal{M} \rightarrow \mathbb{R}$ are

$$
\begin{aligned}
\mathcal{M}_{a} & =V^{-1}((-\infty, a]), \\
\mathcal{M}_{a, b} & =V^{-1}([a, b]) .
\end{aligned}
$$

Let us recall some topological definitions given in $[8,9]$.

Definition 6. A topological space is a $n$-cell if it is homeomorph to $\mathbb{R}^{n}$.

$A$ space $X$ is contractible if it is homotopy equivalent to the one-point space.

$A$ subspace $A$ of $X$ is called a deformation retract of $X$ if there exists a continuous function

$$
h:[0,1] \times X \rightarrow X
$$


such that for all $x \in X, a \in A$

$$
\begin{aligned}
& h(0, x)=x, \\
& h(1, x) \in A, \\
& h(1, a)=a .
\end{aligned}
$$

The $k$-th Betti number of $\mathcal{M}$ denoted by $b_{k}$ is the the rank of the $k$-th homology group $H^{k}(\mathcal{M})$. The Euler Characteristic of $\mathcal{M}$ is defined by

$$
\chi(\mathcal{M})=\sum_{i=1}^{k}(-1)^{k} b_{k} .
$$

For a precise definition of the homology group and a survey about homology theory, the reader may refer to [9, Chapter 2].

\section{Morse Lyapunov functions}

Let us prove a result dedicated to isolated critical points of a smooth Lyapunov functions.

Lemma 7. Suppose that $x_{e}$ is an equilibrium point of the dynamical system $(\mathcal{M}, \varphi)$. If $V: \mathcal{M} \rightarrow \mathbb{R}$ is a differentiable Lyapunov function for the system then $x_{e}$ is the only critical point of $V$.

Proof. Suppose that $V$ contains another critical point $x_{c}$ in the domain of attraction. Due to the definition of a Lyapunov function, we have $\mathcal{L}_{f} V\left(x_{c}\right)=0$. But it contradicts the fact that for a Lyapunov function with a single equilibrium point $x \neq x_{e}, \mathcal{L}_{f} V(x)<0$ for all $x \neq x_{e}$.

Let us recall the Morse Lemma given for instance in [7, Lemma 2.2].

Theorem 8 (Morse Lemma). Let $p \in \mathcal{M}$ be a non-degenerate critical point of a smooth function $V: \mathcal{M} \rightarrow \mathbb{R}$. There exists a local coordinate system $\left(x_{1}, \ldots, x_{n}\right)$ in a neighborhood $\mathcal{N} \subset \mathcal{M}$ of $p$ with $x_{i}(p)=0$ for all $1 \leq i \leq n$ and such that for $x \in \mathcal{N}$,

$$
V(x)=V(p)-x_{1}^{2}-\ldots-x_{i}^{2}+x_{i+1}^{2}+\ldots+x_{n}^{2}
$$

where $i=\operatorname{ind}(V, p)$.

We may now characterize the Morse Lyapunov functions.

Corollary 9. Let $p \in \mathcal{M}$ be an equilibrium point of $(\mathcal{M}, \varphi)$ and $V: \mathcal{M} \rightarrow \mathbb{R}_{\geq 0}$ a Morse Lyapunov function. There exists a local coordinate system $\left(x_{1}, \ldots, x_{n}\right)$ around $p$ such that $V$ is locally the canonical quadratic Lyapunov function

$$
V(x)=x_{1}^{2}+\ldots+x_{n}^{2}
$$

with ind $(V, p)=0$. 
Proof. As $p \in \mathcal{M}$ is an equilibrium point and due to Lemma $7, p \in \mathcal{M}$ is the only critical point of $V$. Moreover, $V$ is supposed to be a Morse function and thus by using Theorem $8, V(x)=V(p)-x_{1}^{2}-\ldots-x_{i}^{2}+x_{i+1}^{2}+\ldots+x_{n}^{2}$. As a Lyapunov function is positive definite, we have the result.

Remark 10. The Morse Lyapunov function plays the role of the canonical quadratic Lyapunov function for systems defined on manifolds.

Let us give an example of Morse functions given in [10, Theorem 6.6].

Example 11. Let $\mathcal{M}$ be manifold in Euclidean space. For almost all $p \in \mathbb{R}^{n}$, the quadratic function $L_{p}: \mathcal{M} \rightarrow \mathbb{R}$ defined by $L_{p}(x)=\|x-p\|^{2}$ is a Morse function.

\section{Level sets of a Lyapunov function}

The following theorem is known as the deformation lemma and is the first Morse theorem. It can be founded in [7, Theorem 3.1].

Theorem 12 (Deformation Lemma). Let $V: \mathcal{M} \rightarrow \mathbb{R}$ be a smooth function and $a, b \in V(\mathcal{M})$ such that $a<b$. Assume that $\mathcal{M}_{a, b}$ is compact and does not contain critical point of $V$, then $\mathcal{M}_{a}$ is diffeomorphic to $\mathcal{M}_{b}$. Moreover, $\mathcal{M}_{a}$ is a deformation retract of $\mathcal{M}_{b}$.

By using Theorem 12, we deduce a result dedicated to dynamical systems with asymptotically stable sets.

Corollary 13. Let $\mathcal{M}$ be a smooth Riemannian manifold. If $\mathcal{M}$ contains a closed invariant asymptotically stable set, then for all $a, b \in V(\mathcal{M}), \mathcal{M}_{a}$ is diffeomorphic to $\mathcal{M}_{b}$ and $\mathcal{M}_{a}$ is a deformation retract of $\mathcal{M}_{b}$ where $V$ is a smooth Lyapunov function.

Proof. If $\mathcal{M}$ is a smooth Riemannian manifold and $\mathcal{K} \subset \mathcal{M}$ a closed invariant asymptotically stable set, there exists a smooth Lyapunov function $V: \mathcal{M} \rightarrow \mathbb{R}_{\geq 0}$ for the dynamical system given in [11, Theorem 3.2] and [12]. As $V$ is proper, $\mathcal{M}_{a, b}$ is compact. Due to Lemma $7, \mathcal{M}_{a, b}$ does not contain critical point of $V$ and the result follows from Theorem 12.

A similar result already exists when $\mathcal{M}=\mathbb{R}^{n}$ (see [4, Theorem 1.2].

\section{Systems with a single critical point}

In this section, we recall some results dedicated to systems with a single critical point. Let us recall the Brown-Stallings lemma which can be founded in [10, Lemma 3].

Theorem 14 (Brown-Stallings Lemma). Let $\mathcal{M}$ be a paracompact manifold such that every compact subset is contained in an open set diffeomorphic to Euclidean space. Then $\mathcal{M}$ itself is diffeomorphic to Euclidean space. 
The Brown-Stallings lemma leads to the following necessary condition of asymptotic stability which can be founded in [4, Theorem 2.2].

Corollary 15. Let $\mathcal{M}$ be a paracompact manifold. The domain of attraction of an asymptotically stable equilibrium point is diffeomorphic to an Euclidean space.

Remark 16. Corollary 15 has been mention in [3, Page 22]. If $\mathcal{M} \subset \mathbb{R}^{n}$, then the domain of attraction is diffeomorphic to $\mathbb{R}^{n}$.

Let us recall the second Morse theorem given in [7, Theorem 3.2].

Theorem 17 (Morse Theorem). Let $V: \mathcal{M} \rightarrow \mathbb{R}$ be a Morse function, $p$ a critical point such that ind $(V, p)=i$ and $c=V(p)$. If there exists $\epsilon>0$ such that $\mathcal{M}_{c-\epsilon, c+\epsilon}$ is compact and does not contain other critical point $p$, then $\mathcal{M}_{c-\epsilon} \cup e_{i}$ is a deformation retract of $\mathcal{M}_{c+\epsilon}$ where $e_{i}$ is an $i-$ cell (in particular, $\mathcal{M}_{c+\epsilon}$ has the homotopy type of $\mathcal{M}_{c-\epsilon}$ with an i-cell attached).

In the case of a single critical point, Theorem 17 implies that the level sets of a Morse Lyapunov function are homotopic. But in the case of an asymptotically stable point of a dynamical system, and thus the existence of a Lyapunov function only, there exists a more precise result which asserts that the domain of attraction is contractible. This result has been given by Sontag in [3, Theorem 21].

Theorem 18 (Sontag Theorem). Let us consider the dynamical system $(\mathcal{M}, \varphi)$ with an equilibrium point $x_{e} \in \mathcal{M}$. Suppose that $x_{e}$ is asymptotically stable. Then the domain of attraction of $x_{e}$, which is given by

$$
\mathcal{A}=\left\{x \in \mathcal{M}: \lim _{t \rightarrow+\infty} \varphi(t, x)=x_{e}\right\}
$$

is contractible.

Theorem 18 remains true for all systems even if $\mathcal{M}$ is a general topological space contrary to Corollary 15 and Theorem 17. It is proved by using the flow

as a deformation retract. However, this result cannot be extended to multi equilibria contrary to Morse theory.

\section{Systems with multi critical points}

The following third Morse theorem given in [7, Theorem 3.5] is a generalization of Theorem 17 to multi critical points. In this case, the manifold has the shape of a CW-complex.

Theorem 19 (Morse Theorem). If $V: \mathcal{M} \rightarrow \mathbb{R}$ is a Morse function such that $\mathcal{M}_{a}$ is compact for each $a \in \mathbb{R}$ then $\mathcal{M}$ has the homotopy type of a $C W$ complex with one $i-$ cell for each critical point of index $i$. 
For more details on CW-complex, the reader may refer to [9].

Corollary 20. Suppose that the dynamical system $(\mathcal{M}, \varphi)$ has several equilibria $\left(x_{1}, \ldots, x_{k}\right)$. If there exists a Morse Lyapunov function $V: \mathcal{M} \rightarrow \mathbb{R}_{\geq 0}$ then $\left\{x_{1}, \ldots, x_{k}\right\}$ is a retract of the domain of attraction.

Proof. Due to Lemma 7 and Theorem $19, \mathcal{M}$ has the homotopy type of $\bigcup_{1<i<k}\left\{e_{i}\right\}$. Moreover, as $V$ is a Lyapunov function, we deduce with Corollary 9 that $\mathcal{M}$ has the homotopy type of $\left\{x_{1}, \ldots, x_{k}\right\}$.

As an application of Theorem 19, there is the theorem of Reeb which can be found in [10, Theorem 1'].

Theorem 21 (Reeb Theorem). Suppose that $\mathcal{M}$ is compact without boundary, if $V: \mathcal{M} \rightarrow \mathbb{R}$ is a smooth function with only two critical points, then $\mathcal{M}$ is homeomorphic to the $n$-sphere $\mathbb{S}^{n}$.

Remark 22. The reader may notice that the critical points are allowed to be degenerate.

Let us apply the Theorem of Reeb to Lyapunov stability of a dynamical system having two equilibria.

Corollary 23. Let $\mathcal{M}$ be a compact manifold without boundary and $\left(x_{1}, x_{2}\right)$ the only two equilibria of the dynamical systems $(\mathcal{M}, \varphi)$. If $\mathcal{I}=\left\{x_{1}, x_{2}\right\}$ is asymptotically stable for the dynamical system $(\mathcal{M}, \varphi)$, then $\mathcal{M}$ is homeomorphic to the $n$-sphere $\mathbb{S}^{n}$.

Proof. There exists a smooth Lyapunov function $V: \mathcal{M} \rightarrow \mathbb{R}_{\geq 0}$ for the dynamical system given in [11, Theorem 3.2] and [12] with only two critical points due to Proposition 7. The Theorem 21 of Reeb leads to the result.

Example 24. As an example, consider the global set stabilization of the spacecraft attitude [13]. In this case, there exists an unwinding phenomenon due to the two equilibria and Theorem 18. But, Corollary 23 is more precise in the case of two equilibria. The system of the spacecraft attitude with two equilibria cannot be locally stabilized when the state space is not homeomorphic to $\mathbb{S}^{n}$.

Theorem 25 (Morse Inequalities). Let $m_{k}$ be the number of critical points of a Morse function $V$ with index $k$, then we have

$$
\begin{aligned}
b_{k} & \leq m_{k} \quad \forall k, \\
\sum_{i=0}^{j}(-1)^{j-i} b_{i} & \leq \sum_{i=0}^{j}(-1)^{j-i} m_{i} \quad \forall j, \\
\chi(\mathcal{M}) & =\sum_{k}(-1)^{k} b_{k}=\sum_{k}(-1)^{k} m_{k} .
\end{aligned}
$$

Let us give a necessary condition for the existence of a Morse Lyapunov function based on the Euler characteristic which is a topological invariant. 
Corollary 26. Let us consider the dynamical system $(\mathcal{M}, \varphi)$ with several equilibria $\left(x_{1}, \ldots, x_{k}\right)$. If there exists a Morse Lyapunov function $V: \mathcal{M} \rightarrow \mathbb{R}_{\geq 0}$ then $\chi(\mathcal{M})=k \geq b_{0}$.

Proof. If there exists a Morse Lyapunov function $V$, due to Lemma 7 and Corollary $9,\left(x_{1}, \ldots, x_{k}\right)$ are the only critical points with indices 0 . Then, by

using Theorem 25, it leads to $\chi(\mathcal{M})=m_{0}=k$. The first inequality of Theorem 25 ensures that $b_{0} \leq m_{0}=k$.

Remark 27. This result implies that if $\chi(\mathcal{M}) \neq k$ then there is no Morse Lyapunov function for the dynamical system. If $\mathcal{M}$ is a compact oriented differentiable manifold, the Euler characteristic can be computed by using the PoincaréHopf Theorem given for instance in [14, Theorem 7.6.5]

\section{Acknowledgment}

The author wishes to thank Pr. Mihai Tibar, from the Department of Mathematics at the University of Lille, for introducing him to Morse theory.

\section{References}

[1] M. Malisoff, M. Krichman, E. Sontag, Global stabilization for systems evolving on manifolds, J. Dynam. Control Systems 12 (2) (2006) 161-184.

[2] L. Rifford, The stabilization problem: AGAS and SRS feedbacks in "Optimal Control, Stabilization and Nonsmooth Analysis", 2004.

[3] E. D. Sontag, Mathematical Control Theory: Deterministic Finite Dimensional Systems, no. 6, Textbooks in Applied Mathematics, Springer-Verlag, New-York, 1998.

[4] F. Wilson, The structure of the level surfaces of a lyapunov function, J. Differential Equations 3 (1967) 323-329.

[5] N. Bhatia, O. Hajek, Local semi-dynamical systems, Lecture Notes in Mathematics, Vol. 90, Springer, 1969.

[6] N. Bhatia, G. Szegő, Stability Theory of Dynamical Systems, Classics in Mathematics, Springer, 2002.

[7] J. Milnor, Morse Theory, Vol. 51, Annals of Mathematics Studies, Princeton University Press, 1963.

[8] E. Bredon, Topology and Geometry, Graduate Texts in Mathematics, vol. 139, Spinger, 1993.

[9] A. Hatcher, Algebraic Topology, Cambridge University Press, 2001. 
[10] J. Milnor, Differential Topology, in "Lectures on Modern Mathematics", Vol. II, Wiley, 1964.

[11] F. Wilson, Smoothing derivatives of functions and applications, Trans. Amer. Math. Soc. 139 (1969) 413-428.

[12] T. Nadzieja, Construction of a smooth lyapunov function for an asymptotically stable set, Czechoslovak Math. J. 40 (2) (1990) 195-199.

[13] S. Li, S. Ding, Q. Li, Global set stabilisation of the spacecraft attitude using finite-time control technique, Internat. J. Control 82 (5) (2009) 822-836.

[14] D. Barden, C. B. Thomas, An Introduction to Differential Manifolds, Imperial College Press, 2003. 